\title{
Combined system usage for large neck anatomy in a high risk abdominal aortic aneurysm patient
}

\author{
Funda Yıldırım¹, Dilşad Amanvermez Şenarslan¹, Adnan Taner Kurdal², Tülün Öztürk, \\ Barış Bayram $^{1}$, Mustafa Cerrahoğlu ${ }^{4}$, İhsan İşkesen ${ }^{4}$, Ömer Tetik ${ }^{4}$
}

\footnotetext{
1) Manisa Celal Bayar University Hafsa Sultan Hospital Cardiovascular Surgery Department, MD., Manisa, Turkey

2) Manisa Celal Bayar University Hafsa Sultan Hospital Cardiovascular Surgery Department, Assist.Prof., Manisa, Turkey

3) Manisa Celal Bayar University Hafsa Sultan Hospital Department of Anesthesiology and Reanimation, Prof.Dr., Manisa, Turkey

4) Manisa Celal Bayar University Hafsa Sultan Hospital Cardiovascular Surgery Department, Prof.Dr., Manisa, Turkey
}

\begin{abstract}
Endovascular aneurysm repair (EVAR) is the first line approach in abdominal aortic aneurysm (AAA) repair. Open surgical repair of AAA is associated with high perioperative mortality and morbidity. A sufficient infrarenal aortic neck is one of the key points for successful outcome after EVAR. We presented in this case report a 75 year- old, male patient with AAA which maximal diameter is $60 \mathrm{~mm}$. The aneurysm had a large neck that presents a hostile neck anatomy. He was unfit for conventional surgery because of comorbidities as chronic obstructive pulmonary disease, newly diagnosed lung cancer and coronary artery disease. He applied to the emergency service with severe and acutely onset back pain and he was admitted to our cardiovascular surgery clinic. Because of acute onset of the back pain, his serious comorbidities and we planned to use of thoracic and abdominal aortic endovascular stent-graft systems indwelling each other which is known as Funnel technique. Despite progress in endovascular therapy, large infrarenal necks remain a challenge for endovascular interventionists. It seems feasible to use combined thoracic and abdominal stent graft as an alternative treatment modality when the patient is not suitable for standard EVAR and open surgery.
\end{abstract}

Key words: Endovascular aneurysm repair, abdominal aortic aneurysm, large neck anatomy.

Yıldırım F., Amanvermez Şenarslan D., Kurdal A. T., Öztürk T., Bayram B., Cerrahoğlu M., İşkesen İ., Tetik Ö. Combined system usage for large neck anatomy in a high risk abdominal aortic aneurysm patient EJCM 2017; 05 (4): 79-82. Doi: 10.15511/ejcm.17.00479. 


\section{Introduction}

Nowadays, endovascular aneurysm repair (EVAR) is the first line approach in abdominal aortic aneurysm (AAA) repair. Open surgical repair of AAA is associated with high perioperative mortality and morbidity. EVAR is a less invasive method than conventional surgery. A sufficient infrarenal aortic neck is one of the key points for successful outcome after EVAR. According to computed tomography angiography (CT), preoperative sizing and planning should be done precisely.(1)

The neck length, angulation, shape, existence of thrombus and calcification should be determined. When the anatomic features of the aneurysm are not favourable for standard EVAR therapies; it is termed "hostile neck anatomy". Proximal neck characteristics remain a challenge in endovascular treatments. Patients with hostile neck anatomy can be managed in some alternative ways; open surgery, chimney technique, fenestrated endovascular grafts and also Funnel technique described by Zachetta et al. ${ }^{(2,3)}$

Fenestrated endovascular abdominal aortic aneurysm repair (FEVAR) and chimney technique are more expensive, off the shelf fenestrated grafts are not available in emergency and in some countries health insur-

Figure 1. AAA with a large neck $(38 \mathrm{~mm}$ internal diameter at just below the renal artery level), axial view of computed tomography anjiography.

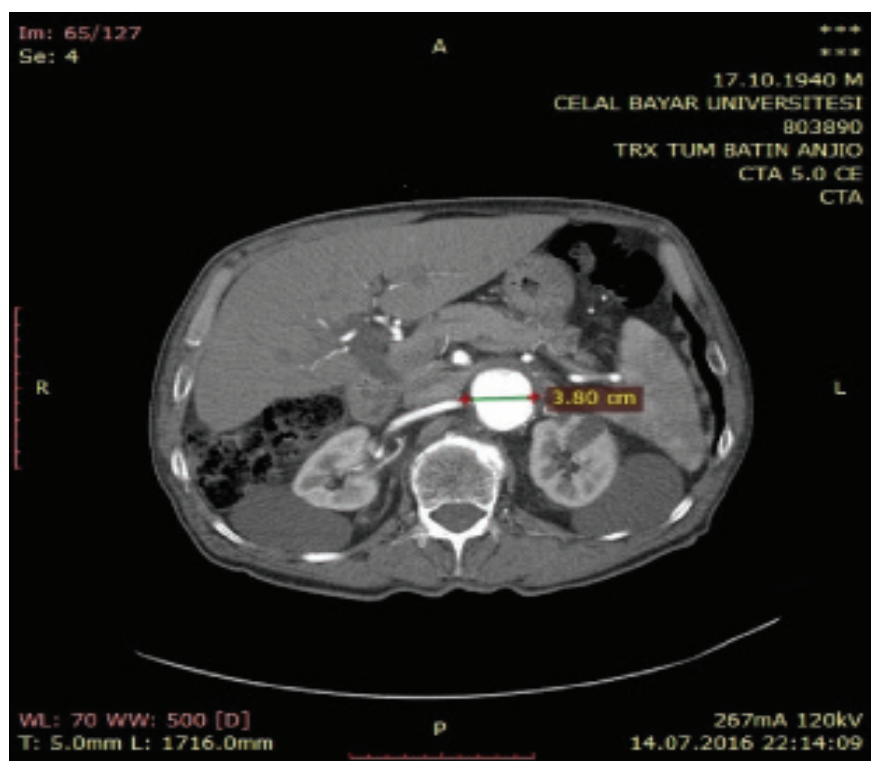

ance may not cover the expense of these advanced techniques. In these circumstances funnel technique in which a thoracic stent-graft is placed through the main body of a bifurcated or uni-iliac endograft, must kept in mind.

\section{Case}

We presented in this case report a 75 year- old, male patient with AAA which maximal diameter is $60 \mathrm{~mm}$. The aneurysm had a large neck that presents a hostile neck anatomy. He was unfit for conventional surgery because of comorbidities as chronic obstructive pulmonary disease, newly diagnosed lung cancer and coronary artery disease. He applied to the emergency service with severe and acutely onset back pain and he was admitted to our cardiovascular surgery clinic.

In the CT Angiographic examination there was no dissection but he had an AAA with a large neck $(38 \mathrm{~mm}$ internal diameter at just below the renal artery level) as shown in Figure 1, 2, 3. Because of acute onset of the back pain, his serious comorbidities and also because of insurance policies of our country we planned to use of thoracic and abdominal aortic endovascular stent-graft systems indwelling each other which is known as Funnel technique.

Figure 2. AAA with a large neck (38 mm internal diameter at just below the renal artery level), coronal view of computed tomography anjiography.

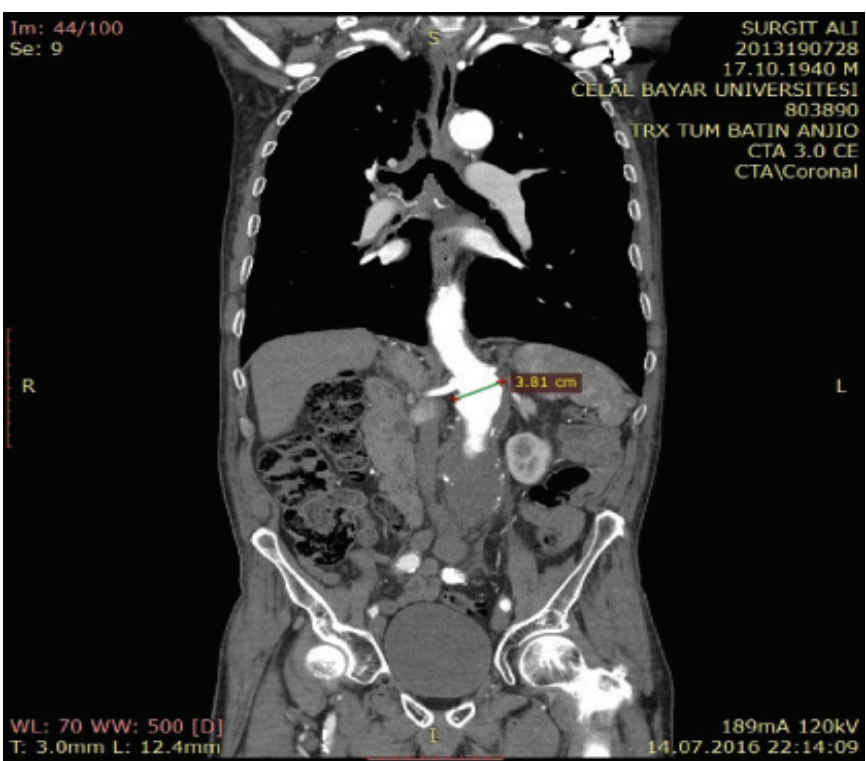


He was treated combining Valiant Captivia ${ }^{\circledR}($ Medtronic, Minneapolis, MN) and Endurant ${ }^{\circledR}$ (Medtronic, Minneapolis, MN) endoprosthesis. We prepared the patient for intervention in hybrid operating room with epidural anaesthesia. Both femoral arteries were surgically exposed for arterial access and snared with purse-string sutures to control bleeding from access sites. Firstly, Valiant Captivia Thoracic Stent Graft ${ }^{\circledR} 44-44 \mathrm{~mm}$ diameter and $100 \mathrm{~mm}$ length stent graft was deployed just below the lowest renal artery. Then Endurant II ${ }^{\circledR}$ aorto-uni-iliac 36-14 mm diameter and $102 \mathrm{~mm}$ length stent-graft was deployed inside of the previous endograft with an overlap of $50 \mathrm{~mm}$.

Later Endurant II® 16-16 mm diameter and $82 \mathrm{~mm}$ length extension stent-graft was inserted to the left iliac arterial system. The right common iliac artery was coiled with endoluminal occluder ${ }^{\circledR}$ (OCL22, Medtronic Cardiovascular, Santa Rosa, CA). The control angiography showed a trace of endoleak from the proximal neck. The Reliant balloon catheter ${ }^{\circledR}$ was used for proximal landing zone fixation. The control angiography didn't show any type I endoleak after ballooning of the proximal neck.

At last, uni-iliac EVAR is accomplished by a left to right femorofemoral bypass operation with an 8-mm ringed PTFE graft. The operation was ended without any trouble. The patient was discharged from the hos-

Figure 3. Preoperative 3-dimensional CT angiography reconstruction; hostile neck and hostile iliac artey anatomy.

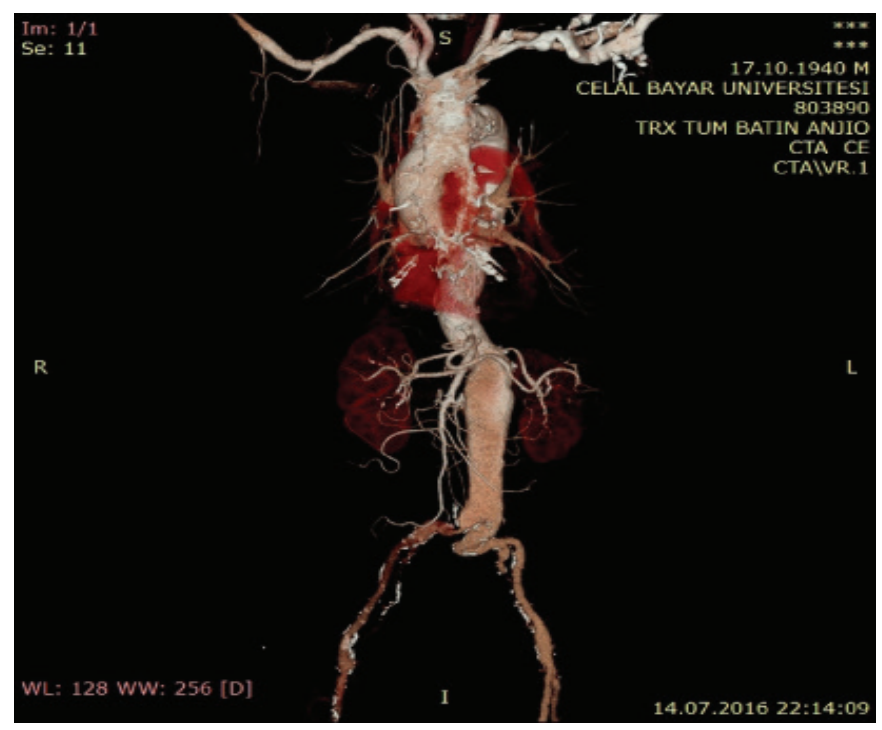

pital at postoperative third day. The patient has still no problem in his first and third month visits.

\section{Discussion}

As many as $20-50 \%$ of AAA patients have anatomy that is not convenient for a standard endovascular therapy. ${ }^{(4)}$ Funnel technique is an alternative way for patients with large hostile neck anatomy who are not suitable for open surgery and the other endovascular methods as FEVAR and chimney grafts. There are limited number of studies related to combined usage of thoracic and abdominal stent-grafts (Funnel Technique). ${ }^{(2-5)}$

Most of the literature consists of case reports. Zachetta et al. first described the method in a case report in 2006. ${ }^{(2)}$ Ronsivalle S. et al. described Funnel technique for EVAR: "a way out" for AAA with ectatic proximal necks. ${ }^{(4)}$ They both reported that the method was secure and effective in sealing to a large aortic neck. They did not observe type I endoleak or migration over a midterm follow up.

Many manufacturers latest-generation abdominal stent grafts have maximal 35-36 mm diameter neck for trunk and ipsilateral leg endoprothesis. But when the patient has a larger neck and given the $10-20 \%$ oversizing ratio for adequate sealing, the interventionist might need additional stents for endovascular treatment.

Secure fixation and adequate sealing zone at the proximal and distal AAA necks are one of the most important factors in predicting a successful outcome of EVAR..$^{(2)}$ Large stent grafts are more at the risk of proximal endoleak and reintervention in the long run. ${ }^{(6,7)}$

The authors emphasized the importance of at least $40 \mathrm{~mm}$ overlapping to the thoracic stent-graft, leaving more than $40 \mathrm{~mm}$ extending beyond the main body of the bifurcated device to ensure expansion to its nominal diameter and adequate sealing. ${ }^{(2,4)}$ In our case, we also obeyed this protocol.

In a recent case report and literature review Pecoraro et al. compared large neck (24-34 mm) AAA treated by standard stent-grafts and wide neck ( $\geq 35 \mathrm{~mm}$ ) AAA treated by Funnel technique. He reports; surprisingly 
higher incidence of migrations in large neck group. Authors interpreted as Funnel technique may be a more valuable deployement methods than we thought. ${ }^{(5)}$

In some clinics, all patients who undergo EVAR are applied sac thrombization with intrasac biomaterials to prevent endoleaks. $^{(2,4)}$ But in our country, it is not possible because health insurance does not cover its expense. So, we did not apply sac thrombization. We have not yet mid-term and long-term follow-up results. We need further follow-up reports to be sure of its durability.

\section{References}

1. De Vries J-PPM. The proximal neck: the remaining barrier to a complete EVAR world. Semin Vasc Surg 2012; 25(4): 182-6.

2. Zanchetta M, Faresin F, Pedon L, Riggi M, Colonna S, Lipari R, et al. Funnel technique for first-line endovascular treatment of an abdominal aortic aneurysm with an ectatic proximal neck. J Endovasc Ther 2006; 13(6): 775-8.

3. Massara M, Prunella R, Gerardi P, De Caridi G, Serra R, Notarstefano $\mathrm{S}$, et al. A Combination of Thoracic and Abdominal Stent-Grafts to Treat An Abdominal Aortic Aneurysm with Hostile Proximal Neck. Ann Vasc Surg 2017; 39: 292.e5-292.e8.

4. Ronsivalle S, Faresin F, Franz F, Rettore C, Zanchetta M, Zonta L. Funnel technique for EVAR: 'a way out' for abdominal aortic aneurysms

\section{Conclusion}

Despite progress in endovascular therapy, large infrarenal necks remain a challenge for endovascular interventionists. Different methods of endovascular therapies like "Funnel technique" enables us to treat high risk, old patients with multiple comorbidities in a short period of hospital stay. It seems feasible to use combined thoracic and abdominal stent graft as an alternative treatment modality when the patient is not suitable for standard EVAR and open surgery. with ectatic proximal necks. Ann Vasc Surg 2012; 26(1): 141-8.

5. Pecoraro F, Pakeliani D, Dinoto E, Bajardi G. Endovascular treatment of large and wide aortic neck: case report and literature review. Gen Thorac Cardiovasc Surg 2017; 65(4): 219-24.

6. Gargiulo M, Gallitto E, Wattez H, Verzini F, Bianchini Massoni C, Loschi D, et al. Outcomes of endovascular aneurysm repair performed in abdominal aortic aneurysms with large infrarenal necks. J Vasc Surg 2017;3:1-8. DOI: 10.1016/j.jvs.2017.01.066. [Epub ahead of print]

7. Kaladji A, Steintmetz E, Gouëffic Y, Bartoli M, Cardon A, Academic Association for Surgical Research (AURC). Long-Term Results of Large Stent Grafts to Treat Abdominal Aortic Aneurysms. Ann Vasc Surg 2015; 29(7): 1416-25.
Received: 16/06/2017

Accepted: 04/11/2017

Published: $15 / 12 / 2017$

Disclosure and conflicts of interest:

The authors declare no conflict of interest.

\section{Corresponding author:}

MD. Funda Yıldırım

Mail: fundanizamoglu@yahoo.com 